\title{
Liver Laceration
}

National Cancer Institute

\section{Source}

National Cancer Institute. Liver Laceration. NCI Thesaurus. Code C50854.

A cut or tear in the liver. 